\title{
PROMIS Pain Interference and Physical Function Scores Correlate With the Foot and Ankle Ability Measure (FAAM) in Patients With Hallux Valgus
}

\author{
Devon C. Nixon MD, Jeremy J. McCormick MD, Jeffrey E. Johnson MD, \\ Sandra E. Klein MD
}

Received: 25 March 2017/Accepted: 7 August 2017/Published online: 23 August 2017

(C) The Association of Bone and Joint Surgeons (B) 2017

\begin{abstract}
Background Traditional patient-reported outcome instruments like the Foot and Ankle Ability Measure (FAAM) quantify patient disability but often are limited by responder burden and incomplete questionnaires. The Patient-Reported Outcome Measurement Information System (PROMIS) overcomes such obstacles through computer-adaptive technology and can capture outcome data from various domains including physical and psychosocial function. Prior work has compared the FAAM with PROMIS physical function; however, there is little evidence comparing the association between foot and ankle-specific tools like the FAAM with more general outcomes measures of PROMIS pain interference and depression in foot and ankle conditions.

Questions/purposes (1) We asked whether there was a relationship between FAAM Activities of Daily Living (ADL) scores with PROMIS physical function, pain interference, and depression in patients with hallux valgus.
\end{abstract}

Each author certifies that neither he or she, nor any member of his or her immediate family, have funding or commercial associations (consultancies, stock ownership, equity interest, patent/licensing arrangements, etc) that might pose a conflict of interest in connection with the submitted article.

All ICMJE Conflict of Interest Forms for authors and Clinical Orthopaedics and Related Research ${ }^{\mathbb{R}}$ editors and board members are on file with the publication and can be viewed on request. Each author certifies that his or her institution approved the human protocol for this investigation and that all investigations were conducted in conformity with ethical principles of research.

D. C. Nixon, J. J. McCormick, J. E. Johnson, S. E. Klein ( $\square)$ Department of Orthopedic Surgery, Washington University School of Medicine, 660 S Euclid Avenue, Campus Box 8233, St Louis, MO 63110, USA

e-mail: kleins@wudosis.wustl.edu
(2) Additionally, we asked if we could identify specific factors that are associated with variance in FAAM and PROMIS physical function scores in patients with hallux valgus.

Methods Eighty-five new patients with either a primary or secondary diagnosis of hallux valgus based on clinic billing codes from July 2015 to February 2016 were retrospectively identified. Patients completed FAAM ADL paper-based surveys and electronic PROMIS questionnaires for physical function, pain interference, and depression from new patient visits at a single time. Spearman rho correlations were performed between FAAM ADL and PROMIS scores. Analyses then were used to identify differences in FAAM ADL and PROMIS physical function measures based on demographic variables. Stepwise linear regressions then determined which demographic and/or outcome variable(s) accounted for the variance in FAAM ADL and PROMIS physical function scores.

Results FAAM scores correlated strongly with PROMIS physical function $(\mathrm{r}=0.70, \mathrm{p}<0.001)$, moderately with PROMIS pain interference $(\mathrm{r}=-0.65, \mathrm{p}<0.001)$, and weakly with PROMIS depression $(\mathrm{r}=-0.35, \mathrm{p}<0.001)$ scores. Regression analyses showed that PROMIS pain interference scores alone were associated with sizeable portions of the variance in FAAM ADL $\left(\mathrm{R}^{2}=0.44, \mathrm{p}<\right.$ $0.001)$ and PROMIS physical function $\left(\mathrm{R}^{2}=0.57, \mathrm{p}<\right.$ $0.001)$ measures.

Conclusions PROMIS function and pain measures correlated with FAAM ADL scores, highlighting the interrelationship of pain and function when assessing outcomes in patients with hallux valgus. PROMIS tools allow for more-efficient data collection across multiple domains and, moving forward, may be better poised to monitor changes in pain and function with time compared with traditional outcome measures like the FAAM. 
Clinical Relevance The relationships shown here between PROMIS and FAAM scores further support the use of PROMIS tools in outcomes-based research. In patients with hallux valgus, pain-related disability appears to be a central feature of the patient-experience. Future studies should assess the association of various outcome domains on other common foot and ankle diagnoses.

\section{Introduction}

Foot and ankle disorders cause substantial patient disability; this then can be quantified through patient-reported outcome (PRO) instruments like the Foot and Ankle Ability Measure (FAAM) [14]. The FAAM originally was validated in a diverse population of patients undergoing physical therapy for lower extremity, foot, or ankle disorders [14] and further validated in athletes with chronic ankle instability [4]. However, traditional tools like the FAAM can be limited by responder burden and incomplete questionnaires [8, 11]. Identifying a universal PRO measure that captures patient morbidity but overcomes these obstacles remains a topic of great interest [12, 13]. The Patient-Reported Outcome Measurement Information System (PROMIS) offers potential in this arena [9, 10]. PROMIS (www.nihpromis.org) uses computer-adaptive technology to precisely collect data regarding various health domains including physical function, pain, and depression in less time and with fewer questions than traditional instruments, like the FAAM, that require completion of a fixed number of questions.

Studies of PROMIS tools in patients with lower extremity disorders have focused primarily on functional disability. Recent work showed comparable reliability and responsiveness between FAAM and PROMIS physical function scores with PROMIS data collected more efficiently and without ceiling effects [8]. Similar work in upper extremity studies has shown that PROMIS function scores strongly correlated with the Quick-Disabilities of the Arm, Shoulder, and Hand (QuickDASH) and the American Shoulder and Elbow Surgeons score [1, 2, 6, 15, 20]. Interestingly, PROMIS pain interference scores were the strongest predictors of functional disability in patients with upper extremity disorders with PROMIS depression outcomes exhibiting less of an effect $[6,15,17]$. No report to date, to the best of our knowledge, has similarly examined the relationship of PROMIS pain interference and depression scores to lower extremity disability. Understanding what areas of disability patients with hallux valgus are experiencing at presentation for care could help with better understanding what features are central to their diagnosis. For example, is hallux valgus primarily a diagnosis defined by functional limitations, pain-related disability, psychosocial deficits (ie, depression), or an interplay of multiple domains? Our aim in this study was to compare FAAM activities of daily living (ADL) scores with PROMIS physical function, pain interference, and depression measures. Specifically, we asked: (1) Do PROMIS physical function, pain interference, and depression scores correlate with the FAAM in patients with hallux valgus? (2) What variables are associated with variance in FAAM and PROMIS physical function scores in patients with hallux valgus?

\section{Patients and Methods}

After institutional review board approval, we retrospectively identified all new patient visits to our foot and ankle clinics from July 2015 to February 2016. Patients with either a primary or secondary diagnosis of hallux valgus were captured using International Classification of Diseases (ICD), 9th Revision (735.0) or ICD, 10th Revision (M20.10, M20.11, M20.12) billing codes. Exclusion criteria included patients younger than 18 years and pregnant women. Demographics were collected by chart review. The presence or absence of comorbidities was based on review of intake questionnaires patients complete before new patient visits. In these surveys, patients can select from approximately 40 potential diagnoses ranging from stroke to depression.

There were 747 new patient visits during our study period based on all possible diagnosis codes. Overall, 131 patients had primary or secondary diagnosis codes of hallux valgus. All patients $(\mathrm{n}=131)$ had complete PROMIS data, but FAAM data were missing or incomplete for 46 patients. Only patients with useable FAAM ADL data were included for further study. Useable FAAM ADL data were defined using prior methodology with a minimum of 19 of the 21 total questions in the FAAM completed accurately [13]. Therefore, 85 patients were identified for analysis (Table 1).

New patients routinely completed intake surveys at their clinic appointment including the paper-based FAAM ADL questionnaire [14]. FAAM ADL surveys were not reviewed for completeness at the time of completion in the clinic. FAAM ADL and PROMIS surveys were not randomized in their order of administration - patients completed the tests in an order of their choosing. Therefore, the high proportion of patients excluded owing to missing or incomplete FAAM data (46 patients, $35 \%$ of the total sample) could be attributable to responder burden given the multiple questionnaires patients are asked to complete. However, there were no differences in age, sex, or PROMIS scores between patients with FAAM data and patients excluded for missing FAAM data (Table 2). 
PROMIS physical function, pain interference, and depression scores were captured using criteria defined by the NIH on the day of the clinic appointment. At our institution, patients completed electronic versions of PROMIS questionnaires that allowed for immediate calculation of a domain score relative to the reference population. With the PROMIS tool, a score of 50 is a population average, and 10point changes represent a single SD change. For negatively worded questions (such as pain interference and depression), a higher PROMIS score represents more of the parameter being measured (that is, greater disability). Conversely, a higher PROMIS physical function score represents better function than the population average. PROMIS surveys are freely available in paper form (www.nihpromis.org);

Table 1. Patient demographic data

\begin{tabular}{lc}
\hline Variable & Data \\
\hline Age $($ years)* & $55(13)$ \\
BMI $\left(\mathrm{kg} / \mathrm{m}^{2}\right)^{*}$ & $27(5)$ \\
Sex & \\
Male & $13(15)$ \\
Female & $72(85)$ \\
Injured extremity ${ }^{\dagger}$ & \\
Right & $36(42)$ \\
Left & $18(21)$ \\
Bilateral & $31(36)$ \\
Outcome measures* & \\
FAAM ADL & $72(22)$ \\
PROMIS-physical function & $44(8)$ \\
PROMIS-pain interference & $58(7)$ \\
PROMIS-depression & $47(10)$ \\
VAS & $5(2)$ \\
Selected comorbidities & \\
Diabetes & \\
Rheumatoid arthritis & $5(6)$ \\
\hline
\end{tabular}

*Mean (SD); ${ }^{\dagger}$ number of patients (\% of total patients); FAAM = Foot and Ankle Ability Measure; ADL = activities of daily living; PROMIS = Patient-Reported Outcome Measurement Information System. however, if using the computer-based version of PROMIS, fees may apply for such costs as data collection, storage, and automated score calculation.

VAS scores also were collected on the intake questionnaires and graded 1 to 10 with 1 being no pain and 10 being the worst pain possible. VAS scores were included in this study as the data represent a more-traditional score of patient-reported pain.

Statistics were performed using SPSS Version 17.0 (SPSS Inc, Chicago, IL, USA). Data were assessed for normality using Shapiro-Wilk testing. Correlations (Spearman rho) were performed between FAAM ADL scores and PROMIS physical function, PROMIS pain interference, PROMIS depression, age, BMI, and VAS scores. Correlations were classified as weak $(0.3 \leq \mathrm{r}<0.5)$, moderate $(0.5 \leq \mathrm{r}<0.7)$, or strong ( $\mathrm{r} \geq 0.7)$. An a priori power analysis determined that a minimum of 84 patients would be needed to detect a correlation (Pearson $r \geq 0.3$ ) at $80 \%$ power with $\alpha$ less than 0.05 . The correlation threshold was selected based on previously published data [17].

Bivariate (Mann-Whitney $U$ test and Kruskal-Wallis) analyses were used to identify differences in FAAM ADL and PROMIS physical function scores based on selected demographic variables (sex, BMI, presence or absence of comorbidities, marital status, employment status, prior foot and/or ankle surgery, and smoking status). Significant ( $\mathrm{p}<$ $0.05)$ variables from correlations and bivariate analyses then were included in a stepwise linear regression to assess which variables accounted for variance in FAAM ADL and PROMIS physical function scores, respectively.

\section{Results}

\section{Correlating FAAM with PROMIS}

We detected a strong correlation between FAAM ADL and PROMIS physical function scores $(\mathrm{r}=0.70, \mathrm{p}<0.001)$, a moderate correlation between FAAM ADL and PROMIS

Table 2. Comparison of study cohort to patients excluded owing to missing FAAM data

\begin{tabular}{llll}
\hline Variable & Study cohort & Missing FAAM data & p Value \\
\hline Number of patients & 85 & 46 & $0.65^{\dagger}$ \\
Age* & $55(13)$ & $54(14)$ & $0.22^{\ddagger}$ \\
Sex (M:F) & $13: 72$ & $11: 35$ & \\
Outcome measures* & & $44(8)$ & $0.73^{\dagger}$ \\
PROMIS-Physical Function & $44(8)$ & $58(6)$ & $0.66^{\dagger}$ \\
PROMIS-Pain Interference & $58(7)$ & $44(8)$ & $0.06^{\dagger}$ \\
PROMIS-Depression & $47(10)$ &
\end{tabular}

${ }^{*}$ Mean (SD); ${ }^{\dagger}$ Student's t-test; ${ }^{*}$ chi-square test; FAAM = Foot and Ankle Ability Measure; PROMIS = Patient-Reported Outcome Measure Information System. 
Table 3. Correlations between PROMIS measures and FAAM scores

\begin{tabular}{|c|c|c|c|c|}
\hline \multirow[t]{2}{*}{ Variable } & \multicolumn{2}{|l|}{ FAAM ADL $*, \dagger$} & \multicolumn{2}{|l|}{ PROMIS-PF $^{\dagger}$} \\
\hline & $\mathrm{r}$ & $\mathrm{p}$ value & $\mathrm{r}$ & $\mathrm{p}$ value \\
\hline Age & $-0.11(-0.32$ to 0.11$)$ & 0.31 & $-0.13(-0.33$ to 0.09$)$ & 0.23 \\
\hline BMI & $-0.28(-0.47$ to -0.07$)$ & 0.01 & $-0.13(-0.33$ to 0.09$)$ & 0.23 \\
\hline FAAM ADL & N/A & N/A & $0.70(0.57-0.79)$ & $<0.001$ \\
\hline PROMIS-pain interference & $-0.65(-0.76$ to -0.51$)$ & $<0.001$ & $-0.76(-0.84$ to -0.65$)$ & $<0.001$ \\
\hline PROMIS-depression & $-0.35(-0.52$ to -0.15$)$ & $<0.001$ & $-0.44(-0.60$ to -0.25$)$ & $<0.001$ \\
\hline PROMIS-physical function & $0.70(0.57-0.79)$ & $<0.001$ & N/A & N/A \\
\hline VAS & $-0.60(-0.73$ to -0.43$)$ & $<0.001$ & $-0.54(-0.68$ to -0.36$)$ & $<0.001$ \\
\hline
\end{tabular}

*Data not normally distributed based on Shapiro-Wilk testing $(\mathrm{p}<0.05) ;{ }^{\dagger}$ Spearman correlation coefficients calculated for all data and displayed along with 95\% CI; PROMIS = Patient-Reported Outcome Measurement Information System; FAAM = Foot and Ankle Ability Measure; ADL = activities of daily living; $\mathrm{PF}=$ physical function; N/A = not applicable.

pain interference scores $(\mathrm{r}=-0.65, \mathrm{p}<0.001)$, and a weak correlation between FAAM ADL and PROMIS depression scores $(\mathrm{r}=-0.35, \mathrm{p}<0.001)$ (Table 3$)$. To clarify, an inverse correlation indicates that decreasing function-as measured by the FAAM ADL-appears to be associated with more pain, and to a lesser degree, more depression as measured by the relevant PROMIS tools. No correlation between FAAM score and patient age was observed $(r=$ $-0.11, \mathrm{p}=0.31$ ), but there was a weak relationship between decreasing FAAM scores and increasing BMI ( $\mathrm{r}=$ $-0.28, \mathrm{p}=0.01)$. There was no correlation between PROMIS physical function score with either patient age or BMI $(\mathrm{r}=-0.13, \mathrm{p}=0.23 ; \mathrm{r}=-0.13, \mathrm{p}=0.23$; respectively). VAS scores correlated with FAAM ADL and PROMIS physical function scores $(\mathrm{r}=-0.60, \mathrm{p}<0.001 ; \mathrm{r}$ $=-0.54, \mathrm{p}<0.001 ; \mathrm{n}=76$, respectively). Bivariate analyses also revealed lower FAAM ADL scores in men ( $\mathrm{p}=$ $0.02)$ and active smokers $(\mathrm{p}=0.03)($ Table 4$)$.

Factors Associated With Variance in FAAM and PROMIS Physical Function Scores

After accounting for potentially confounding variables such as sex, BMI, smoking status, PROMIS pain interference, and PROMIS depression scores, we found that PROMIS pain interference scores alone were associated with $44 \%$ of the variance in FAAM ADL scores $\left(\mathrm{R}^{2}=0.44\right.$, $\mathrm{p}<0.001)$. All other variables were excluded from the final model. Our regression analysis of PROMIS physical function led to similar conclusions. In that model, PROMIS pain interference scores alone were associated with $57 \%$ of
Table 4. Bivariate analyses

\begin{tabular}{|c|c|c|c|c|}
\hline \multirow[t]{2}{*}{ Variable } & \multicolumn{2}{|c|}{ FAAM ADL } & \multicolumn{2}{|c|}{$\begin{array}{l}\text { PROMIS-physical } \\
\text { function }\end{array}$} \\
\hline & $\begin{array}{l}\text { Mean } \\
(\mathrm{SD})\end{array}$ & $\mathrm{p}$ Value & $\begin{array}{l}\text { Mean } \\
\text { (SD) }\end{array}$ & $\mathrm{p}$ Value \\
\hline \multicolumn{5}{|l|}{ Sex $*$} \\
\hline Male $(\mathrm{n}=13)$ & $56(26)$ & 0.02 & $42(11)$ & 0.23 \\
\hline Female $(\mathrm{n}=72)$ & $74(20)$ & & $44(8)$ & \\
\hline \multicolumn{5}{|l|}{ Comorbidities* } \\
\hline No $(n=26)$ & $69(19)$ & 0.35 & $44(9)$ & 0.88 \\
\hline Yes $(n=59)$ & $73(23)$ & & $44(8)$ & \\
\hline \multicolumn{5}{|l|}{ Marital status ${ }^{\dagger}$} \\
\hline Married $(n=63)$ & $72(21)$ & 0.93 & $44(8)$ & 0.87 \\
\hline Divorced $(n=6)$ & $70(26)$ & & $45(7)$ & \\
\hline Single $(\mathrm{n}=11)$ & $72(21)$ & & $45(11)$ & \\
\hline Widowed $(\mathrm{n}=5)$ & $75(32)$ & & $42(13)$ & \\
\hline \multicolumn{5}{|l|}{ Work status ${ }^{\dagger}$} \\
\hline Employed $(\mathrm{n}=58)$ & $73(21)$ & 0.10 & $45(9)$ & 0.23 \\
\hline Disabled $(n=9)$ & $54(29)$ & & $40(11)$ & \\
\hline $\begin{array}{l}\text { Retired or homemaker } \\
(\mathrm{n}=18)\end{array}$ & $75(20)$ & & $43(9)$ & \\
\hline \multicolumn{5}{|l|}{ Prior surgery* } \\
\hline No $(n=57)$ & $73(22)$ & 0.79 & $44(9)$ & 0.75 \\
\hline Yes $(n=28)$ & $70(23)$ & & $44(7)$ & \\
\hline \multicolumn{5}{|l|}{ Smoking status ${ }^{\dagger}$} \\
\hline Never smoked $(n=62)$ & $75(20)$ & 0.03 & $45(8.0)$ & 0.35 \\
\hline Active smoker $(n=6)$ & $52(19)$ & & $44(11)$ & \\
\hline Former smoker $(\mathrm{n}=17)$ & $66(26)$ & & $42(9)$ & \\
\hline
\end{tabular}

*Mann-Whitney U test; ${ }^{\dagger}$ Kruskal-Wallis test; FAAM = Foot and Ankle Ability Measure; ADL = activities of daily living; PROMIS = Patient-Reported Outcome Measurement Information System. 
the variance in PROMIS physical function scores $\left(\mathrm{R}^{2}=\right.$ $0.57, \mathrm{p}<0.001)$.

\section{Discussion}

PROMIS tools allow for outcomes data to be collected more efficiently and with less patient burden as compared with legacy measures like the FAAM [12]. However, limited evidence has directly compared the relationship between PROMIS and the FAAM, and, to the best of our knowledge, no prior studies have compared PROMIS pain interference and depression scores with FAAM ADL for patients with lower extremity disorders. We questioned whether there was a relationship between multiple PROMIS domains (function, pain, and depression) to a traditional measure of lower extremity function (FAAM). We also explored which factors explained the variance in FAAM and PROMIS physical function scores. Our results showed moderate-to-large relationships between FAAM ADL scores and PROMIS physical function and PROMIS pain interference measures in patients with a primary or secondary diagnosis of hallux valgus. There was a weak relationship between FAAM and PROMIS depression scores.

This study had several limitations. First, we selected a common presenting complaint-hallux valgus - to serve as our study population to better understand FAAM and PROMIS scores. Studying only a single, albeit common, diagnosis can be viewed as a strength and a weakness as we attempted to limit the presumed variability that may exist in FAAM ADL and PROMIS scores based on diagnosis. If we had created a heterogeneous study population that included multiple foot and ankle diagnoses, we may have been unable to completely characterize the individual relationships between outcome measures and a common foot and ankle diagnosis like hallux valgus. An additional weakness may be that most of our patients were women (85\%), a proportion comparable to prior studies in hallux valgus $[5,16]$. Women also had higher FAAM ADL scores at initial presentation for hallux valgus care as compared with men; however, there were no sex differences according to PROMIS physical function scores. This suggests that men in our data initially presented to the clinic with greater functional disability-as measured by the FAAM ADL. However, the lack of sex differences according to PROMIS function scores in our study with a small number of male patients $(n=13)$ limits our full interpretation of this sex-specific finding. Our study also only assessed the relationship between a single PROFAAM ADL-to PROMIS measures in patients with hallux valgus, and by doing so, may be a limitation. However, recent work [8] did show stronger correlations between PROMIS physical function to FAAM ADL scores $(\mathrm{r}=0.785)$ than to another commonly used foot and ankle PRO instrument, the Foot Function Index $(r=0.685)$. Furthermore, we used the FAAM ADL in the current study because it has been validated for use in the general population [14], unlike other commonly used outcome tools such as the AOFAS score, which has not been validated or the Foot Function Index, which originally was intended for use in patients with rheumatoid arthritis [3]. Finally, a sizeable portion of our potential patient population was excluded for analysis owing to missing FAAM data. While this could introduce bias, comparisons between our study group and patients with missing FAAM data failed to reveal any differences in age, sex, or PROMIS scores.

The correlation between FAAM ADL and PROMIS physical function in our data was similar in strength to the only prior study directly comparing these measures $(\mathrm{r}=$ 0.785) [8]. The moderate and weak correlations seen here between FAAM to PROMIS pain interference and depression scores, respectively, are similar in strength to upper extremity studies comparing PROMIS tools with the QuickDASH [15, 17]. Menendez et al. [15] showed that QuickDASH scores correlated strongly and weakly with PROMIS pain interference $(\mathrm{r}=0.74)$ and depression $(\mathrm{r}=$ $0.37)$ scores, respectively. Nearly identical correlations were reported by Overbeek et al. [17], with $r=0.74$ and $r=$ 0.34 between QuickDASH to PROMIS pain interference and depression scores. Based on these and our data, it appears that PROMIS pain interference scores have a greater association to upper and lower extremity functional outcomes than PROMIS depression values.

None of the 21 questions in the FAAM ADL survey directly asks patients about pain. However, it appears from our regression data that there may be an underlying relationship between pain-related disability - as measured by the PROMIS pain interference score-and the FAAM. PROMIS pain interference scores alone predicted large portions of the variance for the FAAM ADL and PROMIS physical function scores. Similar findings have been reported in studies of patients with upper extremity disorders $[6,15]$, but to the best of our knowledge, have not been investigated previously in patients with foot and ankle conditions. This concept of pain assessment as an important factor in patients with hallux valgus has been seen previously. Thordarson et al. [19] showed that SF-36 bodily pain scores were worse in patients with bunions as compared with the general population. The interrelationship of pain and function also has been observed outside orthopaedics. Sturgeon et al. [18] revealed that in a large sample of patients treated for chronic pain, higher PROMIS pain intensity scores predicted poorer PROMIS physical function. Our data, similarly, intimates at a complex connection between how patients experience pain and function. 
Our results here of PROMIS pain and function correlating with a traditional measure of lower extremity disabilityFAAM ADL-highlight the interplay between pain and function in orthopaedic foot and ankle conditions with little influence from demographic or psychosocial factors. Given that our study only assessed these measures at a single time, we still need to determine which PROMIS tool(s) - whether that be pain interference or physical function-are best equipped to observe patients after an intervention and monitor clinical improvement. Moving forward, PROMIS physical function and pain scores may be important tools used in counseling and educating patents and ultimately might replace legacy measures like the FAAM that traditionally have been used in foot and ankle outcomes research [7]. Further study on PROMIS instruments in other foot and ankle diagnoses beyond hallux valgus will help provide better understanding of what PROMIS tools are most applicable to each foot and ankle condition.

\section{References}

1. Beckmann JT, Hung M, Bounsanga J, Wylie JD, Granger EK, Tashjian RZ. Psychometric evaluation of the PROMIS Physical Function Computerized Adaptive Test in comparison to the American Shoulder and Elbow Surgeons score and Simple Shoulder Test in patients with rotator cuff disease. J Shoulder Elbow Surg. 2015;24:1961-1967.

2. Beckmann JT, Hung M, Voss MW, Crum AB, Bounsanga J, Tyser AR. Evaluation of the Patient-Reported Outcomes Measurement Information System Upper Extremity Computer Adaptive Test. J Hand Surg Am. 2016;41:739-744.e4.

3. Budiman-Mak E, Conrad KJ, Roach KE. The Foot Function Index: a measure of foot pain and disability. J Clin Epidemiol. 1991;44:561-570.

4. Carcia CR, Martin RL, Drouin JM. Validity of the Foot and Ankle Ability Measure in athletes with chronic ankle instability. $J$ Athl Train. 2008;43:179-183.

5. Coughlin MJ, Jones CP. Hallux valgus: demographics, etiology, and radiographic assessment. Foot Ankle Int. 2007;28:759-777.

6. Doring AC, Nota SP, Hageman MG, Ring DC. Measurement of upper extremity disability using the Patient-Reported Outcomes Measurement Information System. J Hand Surg Am. 2014;39:1160-1165.

7. Ho B, Houck JR, Flemister AS, Ketz J, Oh I, DiGiovanni BF, Baumhauer JF. Preoperative PROMIS scores predict postoperative success in foot and ankle patients. Foot Ankle Int. 2016;37:911-918.

8. Hung M, Baumhauer JF, Brodsky JW, Cheng C, Ellis SJ, Franklin JD, Hon SD, Ishikawa SN, Latt LD, Phisitkul
P, Saltzman CL, SooHoo NF, Hunt KJ; Orthopaedic Foot \& Ankle Outcomes Research (OFAR) of the American Orthopaedic Foot \& Ankle Society (AOFAS). Psychometric comparison of the PROMIS physical function CAT with the FAAM and FFI for measuring patient-reported outcomes. Foot Ankle Int. 2014;35:592-599.

9. Hung M, Baumhauer JF, Latt LD, Saltzman CL, SooHoo NF, Hunt KJ; National Orthopaedic Foot \& Ankle Outcomes Research Network. Validation of PROMIS $\AA$ Physical Function computerized adaptive tests for orthopaedic foot and ankle outcome research. Clin Orthop Relat Res. 2013;471:3466-3474.

10. Hung M, Clegg DO, Greene T, Saltzman CL. Evaluation of the PROMIS physical function item bank in orthopaedic patients. $J$ Orthop Res. 2011;29:947-953.

11. Hung M, Franklin JD, Hon SD, Cheng C, Conrad J, Saltzman CL. Time for a paradigm shift with computerized adaptive testing of general physical function outcomes measurements. Foot Ankle Int. 2014;35:1-7.

12. Hunt KJ, Alexander I, Baumhauer J, Brodsky J, Chiodo C, Daniels T, Davis WH, Deland J, Ellis S, Hung M, Ishikawa SN, Latt LD, Phisitkul P, SooHoo NF, Yang A, Saltzman CL; OFAR (Orthopaedic Foot and Ankle Outcomes Research Network). The Orthopaedic Foot and Ankle Outcomes Research (OFAR) network: feasibility of a multicenter network for patient outcomes assessment in foot and ankle. Foot Ankle Int. 2014;35:847-854.

13. Hunt KJ, Hurwit D. Use of patient-reported outcome measures in foot and ankle research. J Bone Joint Surg Am. 2013;95:e118.

14. Martin RL, Irrgang JJ, Burdett RG, Conti SF, Van Swearingen JM. Evidence of validity for the Foot and Ankle Ability Measure (FAAM). Foot Ankle Int. 2005;26:968-983.

15. Menendez ME, Bot AG, Hageman MG, Neuhaus V, Mudgal CS, Ring D. Computerized adaptive testing of psychological factors: relation to upper-extremity disability. J Bone Joint Surg Am. 2013;95:e149.

16. Nery C, Coughlin MJ, Baumfeld D, Ballerini FJ, Kobata S. Hallux valgus in males: part 1: demographics, etiology, and comparative radiology. Foot Ankle Int. 2013;34:629-635.

17. Overbeek CL, Nota SP, Jayakumar P, Hageman MG, Ring D. The PROMIS physical function correlates with the QuickDASH in patients with upper extremity illness. Clin Orthop Relat Res. 2015;473:311-317.

18. Sturgeon JA, Dixon EA, Darnall BD, Mackey SC. Contributions of physical function and satisfaction with social roles to emotional distress in chronic pain: a Collaborative Health Outcomes Information Registry (CHOIR) study. Pain. 2015;156:26272633.

19. Thordarson DB, Ebramzadeh E, Rudicel SA, Baxter A. Ageadjusted baseline data for women with hallux valgus undergoing corrective surgery. J Bone Joint Surg Am. 2005;87:66-75.

20. Tyser AR, Beckmann J, Franklin JD, Cheng C, Hon SD, Wang A, Hung M. Evaluation of the PROMIS physical function computer adaptive test in the upper extremity. J Hand Surg Am. 2014;39:2047-2051.e4. 